\title{
UMA INTRODUÇÃO A PLACAS LAMINADAS CONSTITUÍDAS DE MATERIAIS COMPOSTOS EM FIBRAS LONGAS
}

\author{
NILSON MAGAGNIN FILHO ${ }^{1}$
}

MAGAGNIN FILHO, N. Uma introdução a placas laminadas constituídas de materiais compostos em fibras longas. Semina: Ci. Exatas/Tecnol. Londrina, v. 21, n. 4, p. 95-108, dez. 2000.

RESUMO: Apresenta-se aqui um texto introdutório e resumido no campo das placas laminadas constituídas de lâminas em materiais compostos de fibras longas. São feitas considerações gerais sobre os materiais compostos relacionadas a seus componentes constitutivos, bem como aos principais materiais utilizados na sua confecção. O comportamento mecânico anisótropo dos compostos em fibras longas é caracterizado, sendo desenvolvidas as expressões de elasticidade linear para a ortotropia, a isotropia transversal e a isotropia completa. Também discorre-se sobre as propriedades termoelásticas de tais materiais, apresentando-se um método simples de determinação dessas propriedades chamado Regra das Misturas. A nomenclatura usada para os laminados em materiais compostos é descrita e em seguida a teoria dos laminados em flexão é apresentada. Por fim, a resistência dos laminados em fibras longas é analisada apresentando-se aí o critério de ruptura de Tsai-Hill e o critério de delaminação de Lee. São apresentadas, também, conclusões sobre a diversidade e a abrangência do campo de estudo em materiais compostos.

PALAVRAS-CHAVE: placas laminadas - estruturas; materiais compostos.

\section{CONSIDERAÇÕES GERAIS SOBRE MATERIAIS COMPOSTOS}

Vinson \& Sierakowski (1987) definem material composto, a grosso modo, como sendo simplesmente o resultado da combinação de dois ou mais materiais para obter um novo material ou uma nova propriedade específica. Entretanto, comentam que tal definição, ao mesmo tempo em que abrangente, pois engloba quaisquer materiais obtidos da reunião de dois ou mais, é também um tanto vaga, pois omite o nível ou escala de caracterização considerada, o que é importante para a escolha da ferramenta analítica a ser utilizada na modelagem teórica de tais materiais. A tal definição acrescentam-se, freqüentemente, então, as palavras "microestrutural" ou "macroestrutural" para definir a escala de caracterização levada em conta na definição do composto. Van Vlack (1970) especifica que, se microestrutural, considera-se a estrutura interna do material com heterogeneidades perceptíveis apenas ao microscópio, enquanto que se macroestrutural, tais heterogeneidades são perceptíveis à vista desarmada.

Entretanto, para propósitos de engenharia, é preciso, ainda, definir mais adequadamente essa classe de materiais. Um olhar sobre os tipos de compostos dos quais a engenharia de estruturas se serve nos leva a caracterizá-los do ponto de vista macroestrutural, pois são, em geral, materiais resultantes da combinação insolúvel de dois ou mais constituintes ou fases. Tais constituintes, como relata Mano (1991), não se dissolvem ou se descaracterizam quando reunidos no composto, formando, cada um, uma parte fisicamente homogênea e, portanto, mantendo suas identidades dentro do novo material. Isso implica em que as heterogeneidades resultantes da união desses dois ou mais materiais diferentes em sua composição ou forma sejam observáveis a um nível macroscópico. Em conseqüência, a caracterização é, portanto, macroestrutural.

As fases constituintes do composto, como relata Mano (1991), são comumente denominadas matriz (ou componente matricial), que é a fase contínua, e reforço (ou componente estrutural), que é a fase descontínua ou dispersa. A função da matriz é envolver o reforço para protegê-lo de danos superficiais, manter sua orientação e espaçamento e transmitir a ele os esforços, que encontrarão no reforço seu principal elemento resistente. Os materiais compostos com

\footnotetext{
${ }^{1}$ Mestre em Engenharia de Estruturas, Universidade Estadual de Londrina, Departamento de Estruturas. Campus Universitário -
} Caixa Postal 6001 . Fone: (43)3714545 e-mail: <magagnin @uel.br>. 
caracterização macrorestrutural constituídos de matriz e reforço, que são os que aqui interessam, utilizam como reforço fibras curtas ou longas, flocos e partículas. Além disso, as componentes podem ser arranjados pela superposição de lâminas, formando os laminados.

Dentre esses tipos de compostos relacionados, de um ponto de vista da aplicação em estruturas, os mais importantes são os filamentares, que são aqueles constituídos por fibras longas ou curtas. Neste trabalho o estudo está restrito aos compostos com fibras longas e laminados, mais especificamente aos compostos constituídos de lâminas com fibras longas com uma ou mais camadas.

As fibras usadas como reforço em compostos são, como discorrem Vinson \& Sierakowski (1987), geralmente, de vidro, boro ou carbono. Para as matrizes utilizam-se os polímeros, como epoxi e poliamida, os metais, como níquel, alumínio e titânio, e as cerâmicas. Os compostos que se utilizam de polímeros como matrizes são chamados poliméricos, os que se utilizam de metais, metálicos, e os que se utilizam das cerâmicas, cerâmicos. As principais combinações entre fibra e matriz são grafite/epoxi, boro/epoxi, carbono/epoxi, kevlar/epoxi, vidro/epoxi, boro/alumínio, grafite/alumínio, sílica/alumínio, urânio/tungstênio, aço/tungstênio, slica/titânio, boro/ titânio e vidro/poliéster. Segundo Mano (1991) as matrizes em epoxi são compatíveis com todos os tipos de fibras e são as mais comumente utilizadas em compostos de alto desempenho, e entre as fibras o destaque é para as de carbono e aramida (Kevlar).

\section{COMPORTAMENTO MECÂNICO DOS COMPOSTOS EM FIBRAS LONGAS}

A caracterização do comportamento mecânico dos materiais ao nível macroscópico, como explicam Vinson'\& Sierakowski (1987), é feita levando em consideração os seguintes tipos fundamentais: homogêneo/isótropo; homogêneo/anisótropo; não homogêneo/isótropo e não homogêneo/anisótropo.

Um material homogêneo é aquele que possui as mesmas propriedades físicas em todos os seus pontos, enquanto que em um material heterogêneo tais propriedades variam a cada ponto. Um material isótropo é aquele que possui, num ponto, as mesmas propriedades elásticas em todas as direções, enquanto que em um material anisótropo tais propriedades variam segundo as direções.

Ainda segundo Vinson \& Sierakowski (1987), os compostos, devido à complexidade envolvida na previsão de seu comportamento, conseqüência de sua própria natureza fibrosa e das heterogeneidades resultantes da combinação de materiais, costumam ser analisados sob dois pontos de vista: micromecânico e macromecânico.

A análise micromecânica reconhece a natureza não homogênea da lâmina do composto, porém ignora a estrutura interna dos constituintes básicos,

- fibra e matriz. Dessa forma, a heterogeneidade da lâmina é reconhecida e levada em conta. No entanto, para desenvolver a metodologia de caracterização da resposta do material nesse nível de análise, várias hipóteses têm de ser introduzidas. A principal delas se refere à geometria do empacotamento das fibras, ou seja, à forma com que as fibras são distribuídas na matriz.

Feita a hipótese do arranjo das fibras, introduzse o conceito de Volume Elementar Representativo que é a menor parte simplificada capaz de representar o domínio completo da lâmina do composto. Tal volume é considerado uniforme, repetido e representativo da resposta característica do composto. A análise matemática pode, então, ser efetuada para relacionar as propriedades e concentrações de fibra e matriz com as propriedades da lâmina do composto, que servirão como valores a serem tomados para a posterior análise macromecânica. Assim, a micromecâníca serve de conexão entre a análise propriamente de engenharia e as teorias que explicam a estrutura e a morfologia da matéria.

A análise macromecânica desconsidera a microestrutura da lâmina e considera somente suas propriedades médias como sendo importantes. Consequentemente, cada lâmina é considerada homogênea e com propriedades diferentes na direção e perpendicularmente à fibra, ou seja, ortótropa. O elemento estrutural é considerado como resultante da superposição de várias lâminas para formar a estrutura desejada, uma viga, uma placa, uma casca, etc. A modelagem matemática do comportamento final da estrutura é feita, então, com as respectivas teorias clássicas existentes, como a teoria clássica de placas e cascas laminadas ortõtropas, por exemplo.

\subsection{Anisotropia Elástica}

\subsubsection{Anisotropia Elástica Linear de um Meio Hiperelástico}

Pode-se escrever a Lei de Hooke generalizada de forma simplificada como:

$$
\sigma_{i}=D_{i j} \varepsilon_{j} \quad(\mathrm{i}, \mathrm{j}=1, \ldots, 6)
$$

Em forma matricial a notação reduzida da Lei de Hooke é: 


$\left[\begin{array}{l}\sigma_{1} \\ \sigma_{2} \\ \sigma_{3} \\ \sigma_{4} \\ \sigma_{5} \\ \sigma_{6}\end{array}\right]=\left[\begin{array}{llllll}D_{11} & D_{12} & D_{13} & D_{14} & D_{15} & D_{16} \\ D_{21} & D_{22} & D_{23} & D_{24} & D_{25} & D_{26} \\ D_{31} & D_{32} & D_{33} & D_{34} & D_{35} & D_{36} \\ D_{41} & D_{42} & D_{43} & D_{44} & D_{45} & D_{46} \\ D_{51} & D_{52} & D_{53} & D_{54} & D_{55} & D_{56} \\ D_{61} & D_{62} & D_{63} & D_{64} & D_{65} & D_{66}\end{array}\right]\left[\begin{array}{l}\varepsilon_{1} \\ \varepsilon_{2} \\ \varepsilon_{3} \\ \varepsilon_{4} \\ \varepsilon_{5} \\ \varepsilon_{6}\end{array}\right]$

Assim sendo, dentro dos limites do regime elástico de Hooke, o comportamento do meio contínuo, homogêneo e anisótropo de um material hiperelástico é caracterizado por 21 coeficientes sendo 18 deles independentes:
$D_{11}, D_{22}, D_{33}, D_{44}, D_{55} \cdot D_{64}, D_{12}=D_{21} \cdot D_{14}=D_{31}, D_{14}=D_{41}$ $D_{15}=D_{51}, D_{16}=D_{63}, D_{23}=D_{32}, D_{24}=D_{42}, D_{25}=D_{52}, D_{26}=D_{62}$. $D_{34}=D_{43}, D_{35}=D_{53}, D_{36}=D_{63}, D_{45}=D_{54}, D_{46}=D_{64}, D_{56}=D_{65}$

A representação matricial $D$ acima descrita é também denominada de matriz de rigidez do material, enquanto que sua inversa, $C=D^{-1}$ é a matriz de flexibilidade.

\subsubsection{Ortotropia}

Os compostos em fibras longas são considerados, de um ponto de vista macromecânico, meios contínuos, homogêneos e anisótropos. Além disso, devido ao arranjo das fibras dentro da matriz eles passam a apresentar planos de simetria elástica, o que reduz seu grau de anisotropia. Esse arranjo obedece, normalmente, a uma disposição simétrica com relação a algum eixo. Devido à existência dessas simetrias o material deixa de ser completamente anisótropo e as matrizes de rigidez e de flexibilidade assumem formas mais simples, quando referenciadas a essas direções.

Em geral os compostos são ortótropos, ou seja, apresentam três planos de simetria ortogonais e com propriedades diferentes segundo cada plano. Para simplificar a análise da ortotropia os eixos coordenados são feitos coincidir com a interseção dos planos de simetria.

Considere-se inicialmente um primeiro plano de simetria, o da lâmina, sendo o plano xy. Assim, a partir dessa primeira condição de simetria, a matriz de rigidez assume a forma simplificada abaixo com somente 13 componentes, das quais 12 são independentes:

$$
D=\left[\begin{array}{cccccc}
D_{11} & D_{12} & D_{13} & 0 & 0 & D_{6} \\
D_{21} & D_{22} & D_{23} & 0 & 0 & D_{26} \\
D_{31} & D_{32} & D_{33} & 0 & 0 & D_{36} \\
0 & 0 & 0 & D_{44} & D_{45} & 0 \\
0 & 0 & 0 & D_{54} & D_{55} & 0 \\
D_{61} & D_{62} & D_{63} & 0 & 0 & D_{66}
\end{array}\right]
$$

Pela consideração de um segundo plano de simetria elástica como sendo o plano xz e também de uma terceira condição de simetria no plano yz (que, porém não impõe novas simplificações) pode-se verificar que, para o material ortótropo, a matriz de rigidez passa a ser expressa por:

$$
D=\left[\begin{array}{cccccc}
D_{11} & D_{12} & D_{13} & 0 & 0 & 0 \\
D_{21} & D_{22} & D_{23} & 0 & 0 & 0 \\
D_{31} & D_{32} & D_{33} & 0 & 0 & 0 \\
0 & 0 & 0 & D_{44} & 0 & 0 \\
0 & 0 & 0 & 0 & D_{55} & 0 \\
0 & 0 & 0 & 0 & 0 & D_{66}
\end{array}\right]
$$

Esta é a matriz de rigidez para um material ortótropo com 9 componentes independentes, pois $D_{12}=D_{21}, D_{13}=D_{31}$ e $D_{23}=D_{32}$.

\subsubsection{Componentes da Matriz de Rigidez Ortótropa}

A fim de facilitar a compreensão do significado físico e a dedução de cada um dos elementos da matriz de rigidez ortótropa analisam-se aqui os componentes da sua inversa, ou seja, da matriz de flexibilidade $\mathrm{C}$.

Analisando-se a matriz de flexibilidade, aos seus componentes podem ser dadas representações obtidas através de analogia com os materiais isótropos, pela realização de ensaios de tração e de cisaihamento.

Pela realização de ensaios de tração simples nas direções $x$ y e $z$ pode-se concluir que:

$$
\begin{array}{lll}
C_{12}=\frac{-v_{y x}}{E_{y}} & C_{22}=\frac{1}{E_{y}} & C_{32}=\frac{-v_{y z}}{E_{y}} \\
C_{13}=\frac{-v_{z x}}{E_{z}} & C_{23}=\frac{-v_{z y}}{E_{z}} & C_{33}=\frac{1}{E_{z}}
\end{array}
$$

Pela realização de ensaios de cisalhamento simples, tendo respectivamente $\tau_{x y} \neq 0, \tau_{y z} \neq 0$ e $\tau_{z x} \neq 0$, obtêm-se: 

$C_{44}=\frac{1}{G_{v z}}$
$C_{55}=\frac{1}{G_{5 x}}$
$C_{66}=\frac{1}{G_{x y}}$

Portanto, a matriz de flexibilidade toma a seguinte forma:

$$
C=\left[\begin{array}{cccccc}
\frac{1}{E_{x}} & \frac{-v_{x y}}{E_{y}} & \frac{v_{x z}}{E_{z}} & 0 & 0 & 0 \\
\frac{-v_{y x}}{E_{x}} & \frac{1}{E_{y}} & \frac{-v_{y z}}{E_{z}} & 0 & 0 & 0 \\
\frac{-v_{z x}}{E_{x}} & \frac{-v_{z y}}{E_{y}} & \frac{1}{E_{z}} & 0 & 0 & 0 \\
0 & 0 & 0 & \frac{1}{G_{y z}} & 0 & 0 \\
0 & 0 & 0 & 0 & \frac{1}{G_{z x}} & 0 \\
0 & 0 & 0 & 0 & 0 & \frac{1}{G_{x y}}
\end{array}\right]
$$

$\operatorname{com} \frac{v_{i j}}{E_{i}}=\frac{v_{j i}}{E_{j}}$.

Assim, para caracterizar um material ortótropo são necessários nove quantidades físicas, ou seja $E_{x x} ; E_{y y} ; E_{z z} ; G_{x y} ; G_{y z} ; G_{z x} ; v_{x y} ; v_{y z} \circ v_{z x}$.

Além disso, vale ressaltar a simetria dos coeficientes elásticos e que a matriz de rigidez é a inversa da de flexibilidade $D=C^{-1}$.

\subsubsection{Isotropia Transversal}

Um outro caso particular é o dos materiais estratificados ou transversalmente isótropos, nos quais as fibras estão dispostas segundo uma única direção. A matriz de flexibilidade passa a ser representada nesse caso como:

$$
C=\left[\begin{array}{cccccc}
\frac{1}{E} & \frac{-v_{x y}}{E} & \frac{-v_{x z}}{E_{z}} & 0 & 0 & 0 \\
\frac{v_{y x}}{E} & \frac{1}{E} & \frac{-v_{y z}}{E_{z}} & 0 & 0 & 0 \\
\frac{-v_{z x}}{E} & \frac{-v_{z y}}{E} & \frac{1}{E_{z}} & 0 & 0 & 0 \\
0 & 0 & 0 & \frac{1}{G_{y z}} & 0 & 0 \\
0 & 0 & 0 & 0 & \frac{1}{G_{z x}} & 0 \\
0 & 0 & 0 & 0 & 0 & \frac{1}{G_{x y}}
\end{array}\right]
$$

Como se vê, nesse caso, têm-se 5 componentes independentes, ou seja, $E, E_{z}, v_{x y}, G_{y z}=G_{x z}$ e $v_{y z}$. Deve-se ainda observar que a isotropia no plano xy induz ainda à relação:

$$
G_{x y}=\frac{E}{2\left(1+v_{x y}\right)}
$$

\subsubsection{Isotropia}

O procedimento para análise da isotropia pode ser encontrado em Malvern (1969). Um material isótropo é aquele que possui as mesmas características em todas as direções, o que implica em que as constantes elásticas sejam independentes da orientação dada aos eixos coordenados.

A matriz de rigidez para um material isótropo pode ser expressa como:

$$
D=\left[\begin{array}{cclccc}
\lambda+2 \mu & \lambda & \lambda & 0 & 0 & 0 \\
\lambda & \lambda+2 \mu & \lambda & 0 & 0 & 0 \\
\lambda & \lambda & \lambda+2 \mu & 0 & 0 & 0 \\
0 & 0 & 0 & \mu & 0 & 0 \\
0 & 0 & 0 & 0 & \mu & 0 \\
0 & 0 & 0 & 0 & 0 & \mu
\end{array}\right]
$$

Como se vê acima, para o caso isótropo a matriz de rigidez é função somente de dois coeficientes elásticos independentes, $\mu$ e $\lambda$, dados por:

$$
\lambda=\frac{E v}{(1+v)(1-2 v)} \quad \mu=\frac{E}{2(1+v)}
$$

\section{PROPRIEDADES TERMOELÁSTICAS DOS COMPOSTOS EM FIBRAS LONGAS}

Ao se efetuar a análise dos compostos laminados como apresentada quando se tratou do comportamento mecânico de tais estruturas deparase com as já conhecidas relações tensãodeformação desses materiais tais como as deduzidas naquela referida seqüencia. As constantes que relacionam tensão e deformação são conhecidas como propriedades termoeiásticas e são características do material utilizado. No caso dos compostos em fibras longas a obtenção de tais propriedades torna-se um tanto complexa quando comparada aos casos dos matérias estruturais de uso corrente, pois naquele caso trata-se de material anisótropo e constituído da combinação de um ou mais materiais diferentes. Além disso, as combinações envolvem, freqüentemente, materiais com propriedades específicas originais de valores relativos muito diferentes (matrizes com propriedades elásticas muito diferentes das das fibras).

McCuliough (1971) destaca que as propriedades 
dos compostos em fibras longas podem ser obtidas, em princípio, a partir de médias das dos constituintes fibra e matriz, levando-se em conta a geometria e a forma da fibra utilizada, a geometria do empacotamento das fibras (ou seja, da disposição das fibras dentro da lâmina do composto), o espaçamento das fibras, a distribuição das cargas na superfície da lâmina e a ligação existente entre a fibra e a matriz. A resposta interna do composto, ou seja, uma certa propriedade, é então determinada tomando-se integrais de volume que relacionam as propriedades da fração de volume dos constituintes do composto com as propriedades médias da lâmina. McCullough (1971) define algumas das propriedades principais a serem determinadas: o Módulo de Elasticidade Longitudinal (E), o Coeficiente de Poisson (v), o Módulo de Elasticidade Transversal (G), o Coeficiente de Expansão Térmica (a) e a Condutividade Térmica (K).

Adams \& Doner (1967) relatam que as propriedades dos compostos podem ser determinadas experimentalmente pela simples realização de ensaios em corpos de prova, porém nos últimos anos têm se desenvolvido métodos de determinação analítica. Tais métodos constituem a chamada análise micromecânica, diferentemente da análise experimental, então dita macromecânica. Aqui fica clara a relação entre a análise micromecânica, já definida anteriormente, com a análise do comportamento final da estrutura. A análise micromecânica estabelece uma forma de determinação das propriedades termoelásticas que vão caracterizar o comportamento do material de que é composta a estrutura final de material composto (viga, casca, placa, etc).

Dentro desse mesmo ponto de vista, Vinson \& Sierakowski (1 987) ressaltam que a micromecânica é introduzida de modo a fornecer uma ferramenta analítica capaz de modelar o comportamento do material composto para que suas propriedades possam ser introduzidas nas equações constitutivas, ou seja, nas relações tensão-deformação. A fim de que isso seja possível várias hipóteses têm de ser introduzidas, tais como:

Hipóteses Relativas ao Comportamento da Fibra: homogeneidade; isotropia; linearidade elástica; espaçamento regular; alinhamento.

Hipóteses Relativas ao Comportamento da Matriz: homogeneidade; isotropia; linearidade elástica.

Hipóteses Relativas ao Comportamento da Lâmina: homogeneidade; ortotropia; linearidade elástica.

McCullough (1971) classifica os métodos de determinação das propriedades termoelásticas dos compostos em quatro tipos básicos: Método Baseado em Conceitos da Resistência dos Materiais; Método Auto-consistente; Método Variacional e Aproximações Numéricas. Tais métodos diferem entre si de acordo com as hipóteses que cada um faz em relação à especificação da geometria dos constituintes fibra e matriz e de acordo com as aproximações feitas com relação à resposta do composto. A seguir fazse uma breve descrição do método mais simples de estimativa das propriedades chamado Regra das Misturas.

\subsection{Regra das misturas}

McCullough (1971) relata que a Regra das Misturas é o modelo mais simples para a previsão das propriedades termoelásticas dos compostos. Nesse modelo a resposta longitudinal do composto a cargas mecânicas, ações térmicas ou elétricas é idealizada como uma reação em paralelo das fibras e da matriz do composto. Para o caso das cargas mecânicas admite-se a hipótese de que as deformações da fibra e da matriz são iguais. Tal modelo conduz a relações lineares simples entre a propriedade do composto e as respectivas propriedades da fibra e da matriz, ponderadas pelas respectivas frações de volume. Uma propriedade qualquer do composto. $P$ é dada por:

$$
P=\sum_{i=1}^{n} v_{i} P_{i}
$$

onde $v_{i}$ é a fração de volume e $P_{i}$ a respectiva propriedade do $i^{e ́ s i m o ~ c o n s t i t u i n t e . ~ P o r ~ e s s a ~}$ expressão vê-se claramente que se a propriedade da fibra $P_{f}$ é muito maior do que a da matriz $P_{m}$, como é o caso frequente em compostos de fibras longas unidirecionais, a contribuição da fibra é que será preponderante na direção longitudinal.

A resposta transversal do composto a essas mesmas cargas é idealizada como uma reação em série das fibras e da matriz do composto. Para 0 caso das cargas mecânicas é assumida a hipótese de deformações equivalentes na fibra e na matriz. Tal modelo também conduz a relações lineares simples entre a propriedade do composto e as respectivas propriedades da fibra e da matriz, ponderadas pelas respectivas frações de volume, mas agora em termos do inverso das propriedades de cada constituinte. Uma propriedade qualquer do composto, $P$ é dada por:

$$
\frac{1}{P}=\sum_{i=1}^{n} \frac{v_{i}}{P_{i}}
$$

onde $v_{i}$ é a fração de volume e $P_{i}$ a respectiva 
propriedade do $i^{\text {esimo }}$ constituinte. Por essa expressäo vê-se claramente que se a propriedade da fibra é muito maior do que a da matriz tem-se $\left(\frac{1}{P_{r}} \leq \frac{1}{P_{n}}\right)$ o que faz concluir que haverá predominância das propriedades da matriz na direção transversal.

McCuliough (1971) comenta que todos os outros modelos teóricos apresentam valores para as propriedades longitudinais que coincidem com os previstos pela Regra das Misturas. Porém, para os valores das propriedades transversais do composto a Regra das Misturas fornece valores excessivamente conservadores. Sendo assim, as propriedades longitudinais dos compostos podem ser previstas com razoável precisão pelas simples equações abaixo:

$$
\begin{gathered}
E_{i}=v_{f} E_{f}+v_{m} E_{m} \\
v_{l t}=v_{f} v_{f}+v_{m} v_{m} \\
\alpha_{l}=\frac{E_{f} \alpha_{f} v_{f}+E_{m} \alpha_{m} v_{m}}{E_{f} v_{f}+E_{m} v_{m}} \\
K_{l}=v_{f} K_{f}+v_{m} K_{m}
\end{gathered}
$$

onde os índices $f$ e $m$ significam fibra e matriz, respectivamente e $E_{l}$ é o módulo de elasticidade longitudinal do composto, $v_{l t}$ é o coeficiente de Poisson no plano do composto, $\alpha_{l}$ é o coeficiente de expansão térmica longitudinal do composto, $K_{l}$ é a condutividade térmica longitudinal do composto, $v_{f}$ e $v_{m}$ são as fraçōes de volume de fibra e matriz, respectivamente, tal que:

$$
v_{f}+v_{m}=1
$$

Tais equações não sugerem qualquer influência de outros parâmetros sobre as propriedades longitudinais do compostos, como forma das fibras, grau de adesão entre fibra e matriz, geometria do empacotamento e espaçamento das fibras, etc, sendo então razoável concluir que tais fatores realmente não exercem significativa influência. Por outro lado, as propriedades transversais são sensíveis a tais fatores, bem como aos modelos e hipóteses utilizados para sua previsão.

Vinson \& Sierakowski (1987) indicam como valores das propriedades transversais de compostos transversalmente isótropos aquelas apresentadas por Hahn (1980). As expressões para as propriedades longitudinais são aquelas previstas pela Regra das Misturas e as para as propriedades transversais são:

$$
\begin{array}{cc}
\frac{1}{G_{12}}=\frac{v_{f}}{G_{12 f}}-\eta_{6} \frac{v_{m}}{v_{f}+\eta_{6} v_{m}} & \frac{1}{G_{23}}=\frac{\frac{v_{f}+\eta_{4} \frac{v_{m}}{G_{m}}}{v_{f}+\eta_{4} v_{m}}}{\frac{1}{K_{\tau}}=\frac{v_{f}}{K_{f}}+\eta_{K} \frac{v_{m}}{K_{m}}} \\
v_{f}+\eta_{K} v_{m} & E_{32}=E_{33}=\frac{4 K_{T} G_{23}}{K_{T}+m G_{23}}
\end{array}
$$

onde os índices $m$ e $f$ significam as respectivas propriedades da matriz e fibra, sendo $G_{12}$ e $G_{23}$ os módulos de elasticidade transversais à fibra nos respectivos planos, $K_{T}$ o módulo de elasticidade volumétrico de deformaçâo no plano e $E_{22}=E_{33}$ os módulos de elasticidade longitudinais perpondiculares às fibras. As constantes $\eta_{6}, \eta_{4}, \eta_{K}, m, K_{m}, K$, e $G_{m}$ säo dadas por:

$$
\begin{array}{cc}
\eta_{\mathrm{t}}=\frac{1+\frac{G_{m}}{G_{12 f}}}{2} & \eta_{4}=\frac{3-\angle v_{m}+\frac{G_{m}}{G_{23 f}}}{4\left(1-v_{m}^{\prime}\right)} \quad \eta_{k}=\frac{1+\frac{G_{w}}{K_{f}}}{2\left(1-v_{m t}\right)} \\
m=1+\frac{4 K_{\mathrm{r}} v_{u}^{2}}{E_{i}} & K_{l}=\frac{E_{f}}{2\left(1-v_{f}\right)} \quad K_{m}=\frac{E_{m}}{2\left(1-v_{m}\right)}
\end{array}
$$

\section{LAMINADOS EM MATERIAIS COMPOSTOS}

\subsection{Tipos e nomenclatura dos laminados}

Os laminados em materiais compostos são formados pela superposição de várias lâminas constituídas, cada uma, de uma matriz envolvendo as fibras longas. Do ponto de vista macromecânico, cada lâmina é considerada homogênea e ortótropa, sendo que o comportamento do laminado depende do número de camadas, ou seja, do número de lâminas superpostas, da espessura e da direção das fibras de cada lâmina. Além disso, a rigidez do laminado resulta da contribuição da rigidez de cada lâmina ou camada.

Um laminado é dito simétrico, de acordo com Prola (1987), quando apresenta simetria geométrica, ou seja, de distribuição de camadas e espessuras, e de propriedades mecânicas em reiação ao seu plano médio.

Segundo Prola (1987), um laminado é dito assimétrico quando apresenta a geometria da distribuição das camadas e as propriedades mecânicas sem qualquer correspondência abaixo e acima da sua superfície média. Um laminado é dito antissimétrico quando apresenta antissimetria geométrica, de distribuição de camadas e espessuras, e de propriedades mecânicas em relação à sua superfície média. 
Prola (1987), além dessa classificação, relata que os laminados podem ser referenciados de acordo com a disposição relativa das fibras em cada lâmina. Particularmente definem-se as seqüências em ângulo, "angle-ply" ou cruzada, "cross-ply". Na seqüência em ângulo, as lâminas são colocadas com orientação de fibras ou direções principais segundo ângulos alternados $+q \quad e \quad-q$ em relação aos eixos do laminado. Os laminados são denominados de seqüência cruzada quando as lâminas forem dispostas com orientação de fibras ou direções principais segundo ângulos altenados $+90^{\circ}$ e $-90^{\circ}$ em relação aos eixos $x$ y no plano do composto.

$\mathrm{Na}$ literatura corrente os laminados são nominados de acordo com sua seqüência de laminação, ou seja, de acordo com os ângulos das fibras que compõem cada camada, em ordem da superior à inferior. Quando as espessuras das lâminas que compõem o laminado forem diferentes umas das outras pode-se incluir na especificação seqüencial o valor da espessura de cada camada. Quando o laminado possui todas as lâminas com mesma espessura é chamado regular.

\subsection{Teoria dos laminados em flexao}

Considere-se um laminado constituído de $\mathrm{N}$ lâminas. Para uma lâmina qualquer $k$ do laminado a Lei de Hooke pode ser expressa como:

$$
\sigma_{i j}^{k}=D_{i j k l}^{k} \varepsilon_{k l}^{k}
$$

O superescrito $k$ é necessário para indicar a orientação, em particular, de cada lâmina com respeito aos eixos $x$ y da placa e sua respectiva matriz de rigidez $D$.

Supondo aqui serem válidas as hipóteses cinemáticas da teoria das placas delgadas (cuja razão entre a espessura e a menor dimensão não ultrapassa 1/5) de que os deslocamentos verticais w são pequenos em relação à espessura e as seções planas permanecem planas após a deformação e ortogonais ao plano médio, podese admitir o seguinte campo de deslocamentos para um ponto a uma distância $z$ do plano médio da placa:

$$
\begin{gathered}
u(x, y, z)=u_{0}(x, y)+z \alpha(x, y) \quad v(x, y, z)=v_{0}(x, y)+z \beta(x, y) \\
w(x, y, z)=w_{0}(x, y)
\end{gathered}
$$

onde $u_{o}, v_{o}$ e $w_{0}$ säo os deslocamentos da superfície média da placa e:

$$
\begin{aligned}
& \bar{\alpha}=\frac{\partial w}{\partial x} \quad \bar{\beta}=\frac{\partial w}{\partial y} \quad \varepsilon_{x}=\frac{\partial u_{0}}{\partial x}+z \frac{\partial \alpha}{\partial x} \quad \varepsilon_{s}=\frac{\partial v_{0}}{\partial y}+z \frac{\partial \beta}{\partial y} \\
& \varepsilon_{z}=0 \quad \varepsilon_{z z}=\frac{1}{2}\left(\alpha+\frac{\partial w}{\partial x}\right) \quad \varepsilon_{x}=\frac{1}{2}\left(\beta+\frac{\partial w}{\partial y}\right) \\
& \varepsilon_{s}=\frac{1}{2}\left(\frac{\partial u_{0}}{\partial y}+\frac{\partial v_{0}}{\partial x}\right)+\frac{z}{2}\left(\frac{\partial \alpha}{\partial y}+\frac{\partial \beta}{\partial x}\right) \\
& \varepsilon_{x_{i}}=\frac{\partial u_{0}}{\partial x} \quad \varepsilon_{x_{0}}=\frac{\partial v_{0}}{\partial y} \quad \varepsilon_{x_{e}}=\frac{1}{2}\left(\frac{\partial u_{0}}{\partial y}+\frac{\partial v_{0}}{\partial x}\right) \\
& k_{x}=\frac{\partial \alpha}{\partial x} \quad k_{y}=\frac{\partial \tilde{\beta}}{\partial y} \quad k_{x y}=\frac{1}{2}\left(\frac{\partial \alpha}{\partial y}+\frac{\partial \hat{\beta}}{\partial x}\right)
\end{aligned}
$$

Levando-se à equação constitutiva, agora incluindo também os possíveis efeitos devidos a variações de temperatura tem-se:

$$
\left[\begin{array}{l}
\sigma_{x} \\
\sigma_{y} \\
\tau_{y z} \\
\tau_{x z} \\
\tau_{x y}
\end{array}\right]_{k}=[D]_{k}\left[\begin{array}{l}
\varepsilon_{x_{0}}+z k_{x}-\alpha_{x} \Delta t \\
\varepsilon_{y_{0}}+z k_{y}-\alpha_{y} \Delta t \\
\varepsilon_{y z} \\
\varepsilon_{x z} \\
\varepsilon_{x y_{0}}+z k_{x y}-\frac{1}{2} \alpha_{x y} \Delta t
\end{array}\right]_{k}
$$

onde $\Delta t$ é a variação de temperatura e $\alpha_{x}$ e $\alpha_{y}$ são os coeficientes de expansão térmica nas direções $x$ e $y$, respectivamente. Os coeficientes de expansão térmica são puramente dilatacionais no sistema 1-2 de coordenadas locais do material, mas devido à rotaçăo para as coordenadas genéricas $\mathrm{x}$-y resulta um $\alpha_{x y}$.

Assim, conhecidas as deformações do plano médio da placa podem ser calculadas as componentes de tensão em cada lâmina que constitui o laminado. Tais tensões são, obviamente, descontínuas na interface entre lâminas, uma vez que cada lâmina possui sua orientação específica.

Em função da variação descontínua das tensões ao longo da espessura do laminado, é mais conveniente trabalhar com suas resultantes em termos de forças e momentos. Definem-se vetores de forças e momentos resultantes das tensões com dimensões de força por unidade de comprimento e momento por unidade de comprimento, respectivamente: 


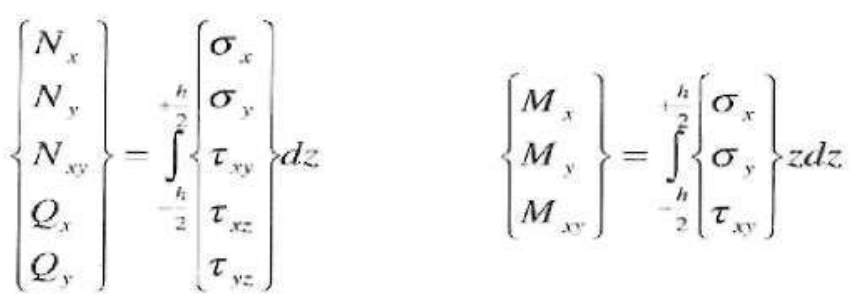

Para uma placa laminada composta por $\mathrm{N}$ camadas ortótropas as resultantes de tensões devem ser obtidas pelo somatório das integrais realizadas em cada lâmina. Assim, pode-se escrever:

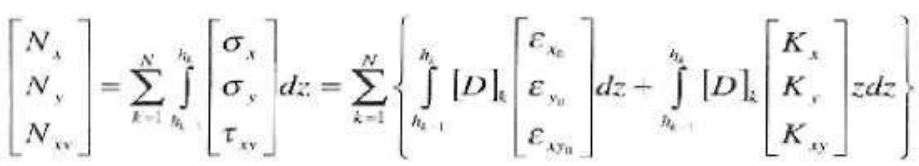

onde, obviamente, utiliza-se somente a porção da matriz de rigidez $[D]_{k}$ requerida para cada integração.

$$
\left[\begin{array}{l}
N_{x} \\
N_{y} \\
N_{x y}
\end{array}\right]=\sum_{k=1}^{N}\left\{[D]_{k}\left[\begin{array}{l}
\varepsilon_{x_{0}} \\
\varepsilon_{y_{0}} \\
\varepsilon_{x y_{0}}
\end{array}\right]_{k}^{h_{h_{k-1}}} d z+[D]_{k}\left[\begin{array}{l}
K_{x} \\
K_{y} \\
K_{x y}
\end{array}\right]_{k} \int_{n_{k-1}}^{h_{k}} z d z\right\}
$$

Em uma forma reduzida pode-se escrever a equação acima como:

$$
[N]=[A]\left[\varepsilon_{0}\right]+[B][K] \quad[M]=[B]\left[\varepsilon_{0}\right]+[D][K]
$$

$\mathrm{Na}$ determinação da resultante das tensões que provocam esforço cortante ( $Q$ ) assume-se que as tensões de cisalhamento variem de forma parabólica através da espessura do laminado e, de acordo com Vinson \& Sierakowski (1987), admite-se para tal uma função contínua dada por:

$$
f(z)=\frac{5}{4}\left[1-\left(\frac{z}{\frac{h}{2}}\right)^{2}\right]
$$

que, levada à integração, fornece:

$Q_{x}=2\left(A_{5 s} \varepsilon_{x z}+A_{45} \varepsilon_{y z}\right) \quad Q_{y}=2\left(A_{45} \varepsilon_{x z}+A_{44} \varepsilon_{y z}\right)$

Podem-se reunir as formas reduzidas apresentadas para [N] e [M] do seguinte modo:
$\left[\begin{array}{l}N_{x} \\ N_{y} \\ N_{x y} \\ --- \\ M_{x} \\ M_{y} \\ M_{x v}\end{array}\right]=\left[\begin{array}{ccccccc}A_{11} & A_{12} & 2 A_{16} & 1 & B_{11} & B_{12} & 2 B_{16} \\ A_{12} & A_{22} & 2 A_{26} & 1 & B_{12} & B_{22} & 2 B_{26} \\ A_{16} & A_{26} & 2 A_{66} & 1 & B_{16} & B_{26} & 2 B_{66} \\ \hdashline B_{11} & B_{12} & 2 B_{16} & 1 & D_{11} & D_{12} & 2 D_{16} \\ B_{12} & B_{22} & 2 B_{26} & 1 & D_{12} & D_{22} & 2 D_{26} \\ B_{16} & B_{26} & 2 B_{66} & \mid & D_{15} & D_{26} & 2 D_{56}\end{array}\right]\left[\begin{array}{l}\varepsilon_{x_{0}} \\ \varepsilon_{y_{0}} \\ \varepsilon_{x_{0}} \\ -- \\ K_{x} \\ K_{y} \\ K_{x}\end{array}\right]$

Analisando a matriz de rigidez acima pode-se dividi-la em três partes segundo as quantidades que eía relaciona. A sub-matriz [A] é a matriz de rigidez extensional, que relaciona as resultantes de tensões no plano [N] com as deformações na superfície média $\left[\mathrm{e}_{0}\right]$. A sub-matriz [D] é a matriz de rigidez flexional que relaciona as tensões binárias $[\mathrm{M}] \mathrm{com}$ as curvaturas $[\mathrm{K}]$. A sub-matriz [B] é chamada matriz de acoplamento, pois relaciona tensões e deformações de diferentes naturezas, ou seja, relaciona momentos [M] a deformações normais [ $\mathrm{e}_{0}$ ] e esforços normais [N] a curvaturas $[\mathrm{K}]$. Isso significa que, no caso geral, no laminado todas as quantidades estáticas e cinemáticas estão relacionadas, ou seja, esforços normais induzem a curvaturas e momentos induzem a deformações normais no plano médio, o que não ocorre nas placas não-laminadas homogêneas, nas quais momentos induzem somente a curvaturas, por exemplo.

Tal acoplamento só é possível de não ocorrer se o laminado possuir simetria completa em relação ao seu plano médio, ou seja, simetria das propriedades elásticas, da orientação e da espessura das lâminas colocadas acima e abaixo da superfície média. Nessas condições a matriz de acoplamento [B] terá todos os seu elementos nulos. Mesmo assim ainda restará o fato de que acoplamento entre alongamento e cisalhamento ocorrerá quando os termos $A_{16}$ e $A_{26}$ forem diferentes de zero, acoplamento entre alongamento e torção ocorrerá quando os termos $B_{16} e B_{26}$ forem diferentes de zero e acoplamento entre flexão e torção ocorrerá quando os termos $D_{16}$ e $D_{26}$ forem diferentes de zero.

Os acoplamentos decorrentes das matrizes $[A]$ e [D] podem ser evitados se, para cada lâmina com orientação q das fibras corresponder uma outra de orientação - q de mesma espessura colocada simetricamente em relação ao plano médio do laminado, pois, nesse caso a soma das contribuições das lâminas para as matrizes [A] e [D] se anulará. Tais laminados são chamados balanceados. Num laminado balanceado desaparecem também os efeitos de interação entre 
momentos fletores e curvaturas de torção, porém, devido ao não obedecimento da simetria os esforços resultantes não serão totalmente desacoplados. Isso só ocorrerá quando se tiver a disposição particular de lâminas orientadas com fibras em ângulos de $0^{\circ}$ e $90^{\circ}$ balanceada, nos chamados laminados cruzados. Em tal caso terse-á o desacoplamento total dos efeitos.

\section{RESISTÊNCIA DOS LAMINADOS EM FIBRAS LONGAS}

Diferentemente das propriedades termoelásticas descritas anteriormente, as propriedades que caracterizam a resistência dos materiais compostos por fibras longas não podem ser tratadas simplesmente como médias de seus elementos constituintes, uma vez que a resistência é um fenômeno de natureza estatística e, portanto, fortemente influenciada por aspectos do comportamento em níveis locais. Em se tratando de materiais constituídos de elementos com diferentes resistências, regiões com resistência inferior à de regiões vizinhas exercerão maior influência na resistência global do composto do que regiões com alta resistência, uma vez que poderá haver ruptura daquela antes desta.

A maneira mais apropriada de levar em consideração tais fenômenos nos materiais compostos é estabelecer aquilo que se convencionou chamar de Mecanismos ou Modos de Ruptura. A identificação de tais modos de ruptura é feita em nível local através de uma análise micromecânica, que leva em consideração o fato de que os elementos constituintes dos compostos, fibra e matriz, têm, em geral, características de resistência muito diferentes uns dos outros. Além disso, a interface entre esses dois elementos básicos apresenta comportamento também diverso dos da fibra e da matriz. Nesse nível de caracterização é possível também levar em conta possíveis falhas e defeitos de fabricação. Assim, identificam-se, de um modo geral, três tipos básicos ou modos de ruptura: ruptura da matriz; ruptura da fibra e ruptura da interface fibra matriz.

Os modos básicos de ruptura são considerados, do ponto de vista da resistência global do laminado, como sendo apenas iniciadores da ruptura global. Para caracterizar a ruptura do elemento estrutural laminado é necessário ainda caracterizar a ruptura da lâmina e do conjunto de lâminas. Uma tal caracterização se dá ao nível não mais da micromecânica, mas da macromecânica ou da análise estrutural.
A caracterização da ruptura da lâmina se dá com base nos conhecidos Critérios de Ruptura e é uma caracterização macroscópica.

5.1 Critérios de ruptura para os laminados em fibras longas

O principal requisito para o projeto de elementos estruturais de materiais compostos é o conhecimento de sua resistência, quando sujeitos a complexas combinações de carregamentos. A resistência e a ruptura de tais materiais pode ser associada com a resistência ao escoamento ou com a resistência última ou de ruptura do material. Para materiais frágeis a resistência última é a mais adequada e para materiais dúcteis a resistência ao escoamento é a mais utilizada. No caso dos compostos em fibras longas, as fibras têm, em geral, comportamento elástico até a ruptura e as matrizes podem ser frágeis ou dúcteis. Além disso, os modos de ruptura já descritos influenciam decisivamente 0 processo de ruptura desses materiais.

Ao nível do elemento estrutural, a caracterização da ruptura é feita com base nos chamandos Critérios de Ruptura. No caso dos materiais compostos em fibras longas, com comportamento anisótropo, esses critérios têm origem nos critérios de ruptura desenvolvidos para os materiais monolíticos. Vinson \& Sierakowski (1987) classificam os critérios como predominante de tensão, predominante de deformação e interativos. O primeiro corresponde ao critério da máxima tensão e o segundo da máxima deformação. Os critérios interativos são os polinômios de tensões.

\subsubsection{Critério de Tsai-Hill}

Hashin (1980) relata que o problema da previsão da ruptura de materiais compostos anisótropos é semelhante ao da construção de um critério de escoamento para um material elastoplástico. Assim, Tsai (1970) admitiu que o critério de ruptura de um composto de fibra unidirecional tem a mesma forma matemática do critério de escoamento de um material idealmente plástico como o proposto por Hill (1950) em que a ruptura é associada à tensão de cisalhamento e insensível à tensão hidrostática.

Vinson \& Sierakowski (1987) descrevem o critério proposto por HILL com a seguinte forma, quando os eixos de anisotropia são os de referência:

$F\left(\sigma_{22}-\sigma_{32}\right)^{2}+G\left(\sigma_{33}-\sigma_{11}\right)^{2}+H\left(\sigma_{11}-\sigma_{22}\right)^{2}+2 L \sigma_{23}^{2}+2 M \sigma_{13}^{2}+2 N \sigma_{12}^{2}=1$ em que os parâmetros $F, C, H, L, M, N$ são característicos do corrente estado de anisotropia. 
Para compostos reforçados unidirecionalmente tem-se $M=N, G=H$ e obtém-se assim:

$F\left(\sigma_{22}-\sigma_{25}\right)^{2}+G\left(\sigma_{33}-\sigma_{11}\right)^{2}+G\left(\sigma_{11}-\sigma_{22}\right)^{2}+2 L \sigma_{23}^{2}+2 M\left(\sigma_{13}+\sigma_{12}\right)^{2}=1$

Para uma lâmina de composto ou laminado em estado plano de tensão tem-se $\sigma_{33}=0$ e obtémse, assim:

$$
F \sigma_{22}^{2}+G \sigma_{11}^{2}+H\left(\sigma_{11}-\sigma_{22}\right)^{2}+2 N \sigma_{12}^{2}=1
$$

Hill (1950) descreve os valores dos parâmetros $F, G, H, L, M, N$ como sendo função das tensões de escoamento à tração $X, Y, Z$ nas direções de simetria elástica e de $R, S, T$ as tensões de escoamento ao cisalhamento com respeito aos mesmos eixos:

$$
\begin{gathered}
\frac{1}{X^{2}}=G+H \quad \frac{1}{Y^{2}}=H+F \quad \frac{1}{Z^{2}}=F+G \quad 2 F=\frac{1}{Y^{2}}+\frac{1}{Z^{2}}+\frac{1}{X^{2}} \\
2 G=\frac{1}{Z^{2}}+\frac{1}{X^{2}}-\frac{1}{Y^{2}} \quad 2 H=\frac{1}{X^{2}}+\frac{1}{Y^{2}}+\frac{1}{Z^{2}} \quad 2 L=\frac{1}{R^{2}} \\
2 M=\frac{1}{S^{2}} \quad 2 N=\frac{1}{T^{2}}
\end{gathered}
$$

Para exemplificar, seja o caso da solicitação plana segundo uma direção qualquer com relação àquela das fibras. Nessas condições o critério se simplifica e pode ser escrito como:

$$
\left(\frac{\sigma_{t}}{X}\right)^{2}+\left(\frac{\sigma_{t}}{Y}\right)^{2}-\frac{\left(\sigma_{t} \sigma_{t}\right)}{X^{2}}+\left(\frac{\tau_{l t}}{T}\right)^{2}=1
$$

Nesse caso tem-se:

$$
\sigma_{t}=\cos ^{2} \theta \sigma_{x} \quad \sigma_{t}=\operatorname{sen}^{2} \theta \sigma_{x} \quad \tau_{t t}=-\operatorname{sen} \theta \cos \theta \sigma_{x}
$$

o que conduz a:

$$
\sigma_{x}=\frac{X}{\left[\cos ^{4} \theta+\frac{X^{2} \operatorname{sen}^{4} \theta}{Y^{2}}+\left(\left(\frac{X^{2}}{T^{2}}\right)-1\right) \cos ^{2} \theta \operatorname{sen}^{2} \theta\right]}
$$

\subsection{Análise da resistência dos laminados}

Os modos de ruptura previstos pela teoria micromecânica servem de ponto de partida para a análise da resistência global dos laminados, uma vez que são considerados os iniciadores da ruptura. Para caracterizar a resistência dos laminados é necessário caracterizar a resistência de cada lâmina e do conjunto de lâminas como um todo, quando sujeitos a complexas condições de carregamento.

A caracterização da resistência da lâmina se dá admitindo que ela está sob um estado plano de tensões. A partir daí tal estado de tensões deve ser determinado para cada lâmina e, com a escolha de um critério de ruptura adequado tal como um daqueles já descritos, verificar a integridade de cada lâmina em todos os seus pontos. A ruptura inicial de uma lâmina é identificada quando o efeito provocado pelo carregamento dado ou caiculado (dependendo do procedimento, se dimensionamento ou verificação do elemento estrutural) excede os limites do critério de ruptura adotado. Quando isso ocorre diz-se que houve a ruptura inicial da primeira lâmina (em inglês "first ply failure", FPF) do laminado. Após ocorrida a ruptura inicial a questão que se coloca é saber se a lâmina inicialmente rompida é capaz de suportar cargas adicionais. Duas hipóteses podem ser feitas. A primeira é desprezar totalmente a camada rompida, considerando que ela já não é capaz de resistir, o que representa uma atitude conservadora em relação à análise da ruptura. A segunda é investigar a causa da ruptura inicial, o que para os compostos em fibra longa representa conhecer o modo de ruptura ocorrido, de tal forma a se desprezar somente a resistência que, de fato, deixou de existir. Assim, se ocorreu a ruptura da matriz em uma camada conhecida, as propriedades de resistência da matriz naquela camada podem ser desprezadas na seqüência da análise. Ao contrário, se ocorreu a ruptura das fibras em uma camada conhecida, as propriedades de resistência das fibras naquela camada podem ser desprezadas na seqüência da análise.

A ruptura de um laminado consistindo de um número de camadas empilhadas com fibras em diferentes orientações ocorre gradualmente. Isso é devido ao fato de que quando uma lâmina se rompe ocorre uma redistribuição das tensões nas lâminas que ainda permanecem intactas. A ruptura final do laminado ocorre quando o elemento estrutural composto do conjunto de lâminas já se degradou o suficiente a tal ponto de não conseguir resistir a cargas adicionais. Quando isso ocorre diz-se que houve a ruptura última (em inglês "last ply failure", LPF) do laminado. Ta! ruptura final pode ocorrer por progressiva degradação das camadas individualmente, rompidas de acordo com os modos de ruptura já descritos, ou por um outro modo de ruptura característico dos laminados, chamado delaminação.

\subsubsection{Análise Progressiva da Ruptura}

A análise dos materiais compostos, devido às características já descritas, em modelos reduzidos ou de protótipos é muito mais difícil do que a análise dos materiais homogêneos e isótropos 
correntes. As técnicas de análise numérica, aliadas à crescente capacidade de memória e velocidade dos computadores tornou possível a análise de elementos estruturais, tais como os laminados reforçados por fibras longas, em detalhes. A técnica de discreíização em elementos finitos vem se destacando entre as técnicas numéricas pela obtenção de resultados muito próximos àqueles do comportamento real de tais estruturas.

Como já foi visto, a ruptura de laminados compostos de fibras longas ocorre de modo progressivo devido à redistribuição de tensões que se dá durante o processo de ruptura. Isso sugere que a análise computacional de tal processo deva ser incrementai, para a simulação da progressão do dano acumulado, e iterativa, para obtenção de configurações de equilíbrio durante o processo de redistribuição das tensões.

Em linhas gerais o processo de análise da ruptura progressiva de laminados em compostos de fibras longas pode ser tal como se descreve a seguir.

Para uma conhecida carga aplicada, as tensões em cada lâmina devem ser calculadas e inseridas no critério de ruptura adotado, tal como aqueles descritos, para determinar se a ruptura ocorreu em cada uma das lâminas (determinar FPF). Se a ruptura não ocorreu, novo incremento de carga pode ser dado e o processo repetido. Se ocorreu a ruptura deve-se procederá identificação do modo de ruptura ocorrido, se da matriz, da fibra ou delaminação. Identificado o modo de ruptura é possível proceder à redução ou eliminação da rigidez correspondente ao modo de ruptura identificado de tal modo a obter nova configuração de equilíbrio com a redistribuição de tensões sem novo incremento de carga. Estabelecida nova configuração de equilíbrio determina-se se a ruptura em qualquer outro ponto de qualquer lâmina ocorreu. Se ocorreu nova ruptura o processo de penalização da rigidez e redistribuição das tensões se repete. Se a ruptura não ocorreu novo incremento de carga pode ser dado. A análise prossegue dessa forma até que a estrutura não seja mais capaz de suportar carga adicional e, nesse instante, diz-se ter ocorrido a ruptura final da estrutura (atingiu-se LPF).

Lee (1982) sugere que, a partir da matriz de rigidez de um elemento finito já conhecida, como abaixo, seja anuíada a rigidez na lâmina do elemento de acordo com o modo de ruptura identificado:

$$
D=\left[\begin{array}{lllccr}
D_{11} & D_{21} & D_{31} & 0 & 0 & 0 \\
D_{12} & D_{22} & D_{23} & 0 & 0 & 0 \\
D_{13} & D_{23} & D_{33} & 0 & 0 & 0 \\
0 & 0 & 0 & D_{23} & 0 & 0 \\
0 & 0 & 0 & 0 & D_{x 1} & 0 \\
0 & 0 & 0 & 0 & 0 & D_{12}
\end{array}\right]
$$

Assim, se o modo de ruptura identificado é da fibra, a matriz de rigidez do elemento é reduzida a zero:

$$
D=[0]
$$

Se o modo de ruptura identificado é da matriz, a matriz de rigidez da lâmina do elemento é reduzida a:

$$
D=\left[\begin{array}{llllll}
D_{11} & 0 & D_{31} & 0 & 0 & 0 \\
0 & 0 & 0 & 0 & 0 & 0 \\
D_{13} & 0 & D_{33} & 0 & 0 & 0 \\
0 & 0 & 0 & 0 & 0 & 0 \\
0 & 0 & 0 & 0 & D_{31} & 0 \\
0 & 0 & 0 & 0 & 0 & 0
\end{array}\right]
$$

o que significa que $\sigma_{T}, \sigma_{T Z}$ e $\sigma_{L Z}$ do elemento serão anulados, sendo $\mathrm{T}, \mathrm{L}$ e $Z$ eixos correspondentes aos eixos $x y z$.

Se o modo de ruptura identificado é de delaminação, a matriz de rigidez de cada elemento adjacente é reduzida a:

$$
D=\left[\begin{array}{lllllc}
D_{11} & D_{21} & 0 & 0 & 0 & 0 \\
D_{12} & D_{22} & 0 & 0 & 0 & 0 \\
0 & 0 & 0 & 0 & 0 & 0 \\
0 & 0 & 0 & 0 & 0 & 0 \\
0 & 0 & 0 & 0 & 0 & 0 \\
0 & 0 & 0 & 0 & 0 & D_{12}
\end{array}\right]
$$

o que significa que $\sigma_{Z Z}, \sigma_{T Z}$ e $\sigma_{Z L}$ do elemento seráo anulados, sendo $T, L$ e $Z$ eixos correspondentes aos eixos $x y z$. 


\section{CONCLUSÕES}

O objetivo geral desse trabalho, de elaborar um texto introdutório no campo das placas laminadas em compostos de fibras longas, foi atingido. O texto apresentado é abrangente, dando uma visão geral dos principais aspectos que envolvem essa nova classe de materiais em desenvolvimento.

Foram apresentadas a definição mais apropriada para os materiais compostos de fibras longas, bem como algumas propriedades dos materiais que são utilizados na sua confecção, evidenciando-se, a partir dessas prorpriedades individuais, o enorme potencial das placas compostas de fibras e matriz, uma vez que tais materiais possuem grande rigidez com baixo peso quando comparados aos materiais de uso corrente mais eficientes. Porém, é também evidente que as desvantagens relacionadas aos compostos estão ligadas aos custos.

Mostrou-se, no que se refere ao comportamento mecânico dos compostos em fibras longas, a importância da análise micromecânica e sua relação com a análise macromecânica, que é a análise mais apropriada para a engenharia. A teoria da anisotropia elástica foi desenvolvida, partindo-se do caso geral de anisotropia para o particular de isotropia completa do material, passando-se pela ortotropia e pela isotropia transversal, que são aplicáveis às placas em materiais compostos.

No tocante à determinação das propriedades termoelásticas dos compostos em fibras longas discorreu-se sobre um método utilizado, apresentandose algumas expressões obtidas pela conhecida Regra das Misturas, um método mais simples de obtenção de tais propriedades baseado na ponderação das propriedades de fibra e matriz pelas respectivas frações de volume. Tal método é aplicável com boa precisão às propriedades longitudinais.

Também classificaram-se os laminados de acordo com sua seqüência de empilhamento e desenvolveu-se a teoria dos laminados quando sujeitos à flexão, inclufndo-se aí os efeitos da variação de temperatura. Conclusões foram tiradas quanto ao acoplamento das quantidades estáticas e cinemáticas para o caso geral e partícularizadas para os casos em que tais acoplamentos podem deixar de existir. No caso geral todas essas quantidades estarão relacionadas e no caso particular dos laminados balanceados com camadas com fibras em zero e noventa graus tal acoplamento desaparecerá.

A resistência dos laminados também foi analisada. Foram apresentados os modos básicos de ruptura da fibra, da matriz e da interface entre ambas, bem como alguns aspectos sobre os critérios de ruptura mais utilizados para a análise da resistência dos compostos em fibras longas. O critério deTsai-Hill foi apresentado e o processo da análise progressiva da ruptura dos laminados foi descrito, apresentando-se aí também um critério de delaminação, o de Lee.

Como se vê pelo texto apresentado a diversidade e a abrangência das possibilidades de aplicação e investigação no campo dos materiais compostos e, particularmente nos de fibras longas, é enorme. Relacionados ao tema estão as aplicações nos campos das engenharias mecânica e civil, bem como aeoroespacial, aeronáutica e automobilística. Os métodos de caracterização de tais materiais também constituem um vasto campo de investigação, bem como aqueles relativos à determinação de suas propriedades e à previsão de sua ruptura.

No campo da Mecânica dos Materiais a investigação sobre os materiais compostos se direciona à formulação dos modelos constitutivos aplicáves a essa categoria de materiais, envolvendo o estudo de sua microestrutura, a simulação dos fenômenos de deformação e ruptura, a formulação termodinâmica e também a análise probabilística da ruptura em decorrência da existência de defeitos de fabricação.

Já no campo da Mecânica das Estruturas, que tem como base as teorias contínuas, envolvendo caracterização macroscópica, a investigação se dá sobre a anisotropia, as grandes deformações, o estudo dos fenômenos acoplados, dos critérios de ruptura e da deterioração por danificação progressiva.

Particularmente no campo da Engenharia Civil desenvolvem-se estudos e aplicações em recuperação e reforço estrutural e também na aplicação aos concretos especiais, particularmente concretos com fibras. 
MAGAGNIN FILHO, N. An introduction to laminated plates consisting of long fiber composite materials. Semina: Ci. Exatas/Tecnol. Londrina, v. 21, n. 4, p. 95-108, dez. 2000.

ABSTRACT: An introductory and brief text about the long fiber composite materials in the laminated plates field is presented. General considerations are made about composite materials related to its constitutive components, as well as the main materials used in its building. The anisotropic mechanical behavior of long fiber composite materials is characterized, and the linear elasticity expression are developed to orthotropy, transversal isotropy and full isotropy. Thermoelastic properties from these materials are also presented and a simple method to its determination, called Rule of Mixtures, is shown. The laminated composite materials nomenclature is described and the bending laminated theory is also shown. At the end, long fiber laminated composite strength is analysed and the Tsai-Hill failure criterion and the Lee delamination criterion are presented. Conclusions about diversity and wide range of composite materials study, are reached.

KEY WORDS: laminated plates - structures; composite materials.

\section{REFERÊNCIAS BIBLIOGRÁFICAS}

ADAMS, D. F. ; DONER, D. R. Longitudinal Shear Loading of a Unidirectional Com positc. Journal of Composite Materials; v.1, p.4-17, 1967.

Transverse Normai Loading of a Uniairectional Composite. Journal of Composite Materia/s, v.1, p.152-164, 1967.

HASHIN, Z. Failure Criteria for Unidirectional Fiber Composites. Journal of Applied Mechanics, v.47, p.329-334, 1980.

HAHN, H. T. Simplified Formulas for Elastic Moduli of Unidirectional Continuous Fiber Composites. Composites Technology Review, Fall 1980.

HILL, R. The mathematical theory of plasticity. London: Oxford University Press, 1950.

LEE, J. D. Three-Dimensional Finite Element Anaysis of Damage Accumulation in Composite Laminate. Computers and Structures, v.15, n.3, p.335-350, 1982.

MALVERN, L. E. Introduction to the Mechanics of a Continuous Medium. [S. I.]: Prentice-Hall, 1969.

MANO, E. B. Polimeros como Materiais de Engenharia. São Paulo: Edgard Blücher, 1991.

McCUllough, R. L. Concepts of Fibor-Resin Composites. New York: Marcel Dekkor, 1971.

PROLA, L. C. Comportamento de Pós-flambagem de Placas Laminadas de Materiais Compostos Dispostos Simetricamente. 1987. Dissertação (Mestrado) - Escola de Engenharia da Universidade Federal do Rio Grande do Sul, Porto Alegre, 1987.

TSAI. S. W.; WU, E. M. A General Theory of Strength for Anisotropic Materials. Journal of Composite Materials, v.5, p.58$80,1971$.

VAN VLACK, L. H. Principios de ciência dos materiais. São Paulo: Edgard Blücler, 1970.

VINSON, J. R.; SIERAKOVSKI, R. L. The Bchavior of Structures Composed of Composite Matcrials. Dordrecht: Martinus Nijhoff Publishers, 1987.

\section{BIBLIOGRAFIA COMPLEMENTAR CONSULTADA}

ABOLIN'SH, D. S. Compliance Tensor for an Elastic Material Reinforced in One Direction. Mekhanika Polimerov, v.1, n.4, p.52-59, 1965.

AGARWAL, B. D.; BROUTMAN, L. J. Analysis and Performance of Fiber Composites. [S. I.]: John Wiley and Sons, 1990.

FOSTER, J. M.; SMITH, C. B.; VACHON, R. I. On Predicting Thermal Conductivity of a Binary Mixture of Solids. Journal of Spacecraft and Rockets, v.3, n.2, p.287-288, 1966.

HASHIN, Z.; ROSEN, B. W. The Elastic Moduli of Fiber Reinforced Materials. Journal of Applied Mechanics, v.31, p.223-232, 1964.

HASHIN, Z. On Elastic Behavior of Fiber-Reinforced Materials of Arbitrary Transverse Phase Geometry. Journal of Mechanics and Physics of Solids, v.13, p.119-134, 1965.

Analysis of Properties of Fiber Composites with Anisotropic Constituints. Journal of Applied Mechanics, v.46, p.543-550, 1979.

HAUG, E.A.R. ; DOWLATYARI, P. Delamination Criterion Identification for Multilayered Composite Laminates. p.336-355, 1991.

HILL, R. Theory of Mechanical Properties of Fibre-Strengthened Materials - III. Self-Consistent Model. Joumal of Composite Materials, v. 13, p.189-198, 1965.

HINTON, E.; OWEN, D.R.J. Finite Element Software for Plates and Shells. Swansea, Pineridge Press, 1984.

HILL, R. Theory of Mechanical Properties of Fibre-Strengthened Materials - III. Self-Consistent Model. Journal of Composite Materials, v.13, p.189-198, 1965.

MULLIN, J.; BERRY, J. M.; GATTI, A. Some Fundamental Mechanisms Applicable to Advanced Filament Reinforced Composites. Journal of Composite Materials, v.2, n.1, p.82103, 1968. 
NOOR. A. K. Mechanics of Anisotropic Plates and Shells - A New Look at an Old Subject. Computers and Structures, v.44, n.3, p.499-514, 1992.

REDDY, J.N. ; PANDEY, A.K. A First-Play Failure Analysis of Composite Laminates. Computers and Structures, v.25, n.3, p. $371-393,1987$.

ROSEN, B.W. Mechanics of Composite Strengthening. Fiber Composito Matorials. Ohio: American Socicty for Metals, 1965.

ROTSCHILDS, R. J.; GILLESPIE, R. J.; CARLSSON, L. A. Instability-Related Delamination Growth in Thermoset and Thermoplastic Composites. Composite Materials.
TOLSON, S. ; ZABARAS, N. Finite Element Analysis of Progressive Failure in Laminated Composite Plates. Computers ans Structures, v.38, n.3, p.361-376, 1991.

WHITNEY, J. M. ; RILEY, M. B. Elastic Properties of Fiber Reinforced Composite Materials. AlAA Journal, v.4, n.9, p.15371542, 1966.

WHITNEY, J. M. Elastic Moduli of Unidirectional Composites with Arisotropic Filaments. Joumal of Composite Materials, v.1, p.188-193, 1967. 